\title{
Early neoplastic and metastatic mammary tumours of transgenic mice detected by 5-aminolevulinic acid-stimulated protoporphyrin IX accumulation
}

\author{
AM Dorward', KS Fancher', TM Duffy', WG Beamer' and H Walt*,2 \\ 'The Jackson Laboratory, 600 Main St Bar Harbor, ME 04609, USA; ${ }^{2}$ Research Division of Gynecology, Department of Obstetrics and Gynecology, \\ University Hospital Zurich, Frauenklinikstrasse 10, $\mathrm{CH}-809$ I Zurich, Switzerland
}

\begin{abstract}
A photodynamic technique for human breast cancer detection founded upon the ability of tumour cells to rapidly accumulate the fluorescent product protoporphyrin IX (PpIX) has been applied to transgenic mouse models of mammary tumorigenesis. A major goal of this investigation was to determine whether mouse mammary tumours are reliable models of human disease in terms of PpIX accumulation, for future mechanistic and therapeutic studies. The haeme substrate 5 -aminolevulinic acid (5-ALA) $\left(200 \mathrm{mg} \mathrm{kg}^{-1}\right)$ was administered to mouse strains that develop mammary tumours of various histological subtypes upon expression of the transgenic oncogenes HRAS, Polyoma Virus middle T antigen, or Simian Virus 40 large T antigen in the mammary gland. Early neoplastic lesions, primary tumours and metastases showed consistent and rapid PpIX accumulation compared to the normal surrounding tissues, as evidenced by red fluorescence $(635 \mathrm{~nm})$ when the tumours were directly illuminated with blue light $(380-440 \mathrm{~nm})$. Detection of mouse mammary tumours at the stage of ductal carcinoma in situ by red fluorescence emissions suggests that enhanced PpIX synthesis is a good marker for early tumorigenic processes in the mammary gland. We propose the mouse models provide an ideal experimental system for further investigation of the early diagnostic and therapeutic potential of 5-ALA-stimulated PpIX accumulation in human breast cancer patients.
\end{abstract}

British Journal of Cancer (2005) 93, I |37- | |43. doi: | 0.1038/sj.bjc.6602840 www.bjcancer.com

Published online 25 October 2005

(c) 2005 Cancer Research UK

Keywords: photodynamic detection; 5-aminolevulinic acid; protoporphyrin IX; mammary tumour; transgenic mice

Synthesis of the fundamental co-factor haeme occurs as a multistep biochemical pathway within the cytoplasmic and mitochondrial compartments of all mammalian cells. Haeme synthesis shows controlled and complex regulation with prominent substrate inhibition at the first step of 5-aminolevulinic acid (5-ALA) production (Ponka, 1999). Exogenous administration of 5-ALA overrides product inhibition, and leads to increased synthesis of the haeme precursors. It is well documented that neoplastic cells derived from multiple human tumour types show an exaggerated capacity for haeme precursor synthesis following 5-ALA exposure relative to normal cells, although the exact contribution to tumour development remains unclear (Collaud et al, 2004). Tumours of the skin, bladder, oesophagus, oral cavity, colon, breast, ovary, cervix, leukocytes and brain accumulate the fluorescent haeme precursor protoporphyrin IX (PpIX) following 5-ALA administration (Stummer et al, 1998; af Klinteberg et al, 1999; Chen et al, 2001; Ladner et al, 2001; Moesta et al, 2001; Betz et al, 2002; Bogaards et al, 2002; Olivo et al, 2003; Stepinac et al, 2003; Löning et al, 2004). In the case of human colon cancers and their metastases, it was observed that PpIX concentrations were elevated compared to the normal surrounding tissues without exogenous 5-ALA administration (Moesta et al, 2001). Investigations to address the mechanism of enhanced PpIX synthesis have

\section{*Correspondence: Professor H Walt; E-mail: heinrich.walt@usz.ch}

Revised 5 August 2005; accepted 27 September 2005; published online 25 October 2005 focussed on the activity of the haeme synthesis enzymes, and suggest that increased activity of the enzymes involved in porphyrin synthesis - 5-ALA dehydratase and hydroxymethylbilane synthase - combined with reduced activity of ferrochelatase to generate haeme from PpIX, may contribute to the net PpIX accumulation response of tumours (Navone et al, 1990; Hinnen et al, 1998; Krieg et al, 2002). Additional factors that influence PpIX accumulation may relate to the tumour micro-environment ( $\mathrm{pH}$, vascularisation) or other properties of tumour cells that could impact upon haeme synthesis, such as altered mitochondrial function, or transport of 5-ALA, porphyrin intermediates, and iron (Collaud et al, 2004).

The general property of PpIX loading by tumour cells, either inherently, or following 5-ALA dosing, has stimulated numerous basic research and clinical studies for potential therapeutic and diagnostic uses. PpIX is a natural photosensitiser that generates reactive oxygen intermediates following excitation with $635 \mathrm{~nm}$ light. Thus 5-ALA-stimulated PpIX loading has been evaluated for photodynamic therapy (PDT) to eradicate tumour cells (Peng et al, 1992; Hua et al, 1995; Casas et al, 2001; Otake et al, 2003). Similarly, PpIX loading stimulated by administration of 5-ALA was investigated for the purpose of photodynamic detection (PDD) of tumour cells (Bedwell et al, 1992; Van Hillegersberg et al, 1992). This strategy utilises blue light excitation $(380-440 \mathrm{~nm})$ to induce red fluorescent emissions $(635 \mathrm{~nm})$ from the tumour cells that concentrate PpIX, and has been put to practical use to identify margins of neoplasia during exploratory or tumour de-bulking 

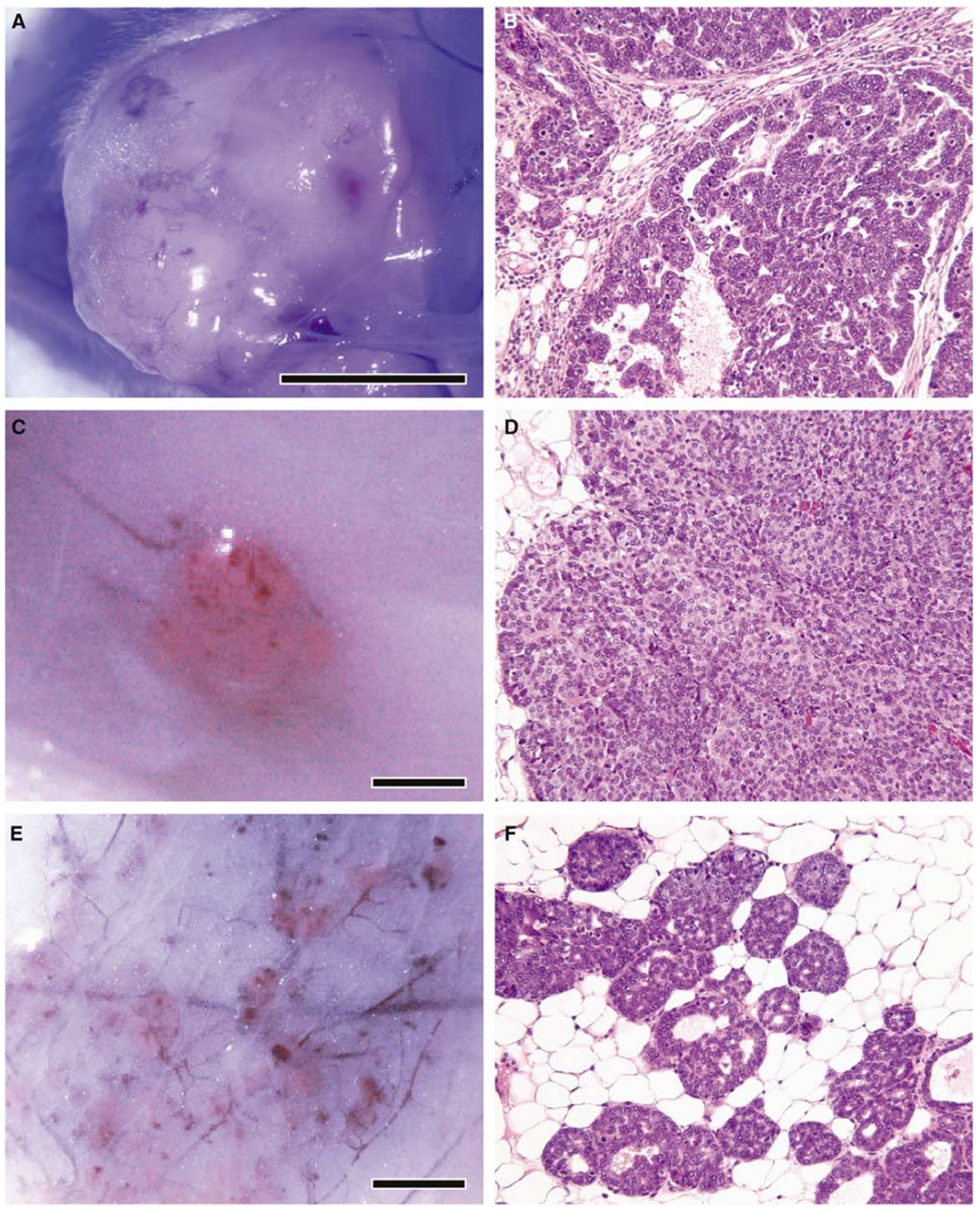

Figure I Digital image capture of PpIX-stimulated red fluorescence in tumours of three mammary tumour-bearing transgenic strains I h following in vivo administration of $200 \mathrm{mg} \mathrm{kg}^{-1}$ ALA, with corresponding H\&E stained histological sections. (A, B) Red fluorescence in a papillary adenocarcinoma of a WapTag I female $(77$ weeks) $(20 \times)$. (C, D) HRAS male mammary tumour (I I weeks) showing prominent fluorescence and solid carcinoma histology $(20 \times)$; (E, F) Fluorescent multifocal tumours of a PyVT female $(5$ weeks) with solid carcinoma histology $(20 \times)$. Scale bar represents I mm. 
Table I Group size and histological characteristics of transgenic strain mammary tumours examined for PpIX accumulation

\begin{tabular}{lccl}
\hline Strain & $\begin{array}{c}\text { Gender } \\
\text { (M/F) }\end{array}$ & $\begin{array}{c}\text { Group } \\
\text { size }(\boldsymbol{n})\end{array}$ & Histological tumour type \\
\hline HRAS & M & 20 & Solid adenocarcinoma \\
PyVT & $F$ & 20 & $\begin{array}{l}\text { Multifocal, solid adenocarcinoma } \\
\text { WapTag| }\end{array}$ \\
& $F$ & 8 & $\begin{array}{l}\text { Papillary adenocarcinoma, glandular/ } \\
\text { solid adenocarcinoma }\end{array}$ \\
\hline
\end{tabular}
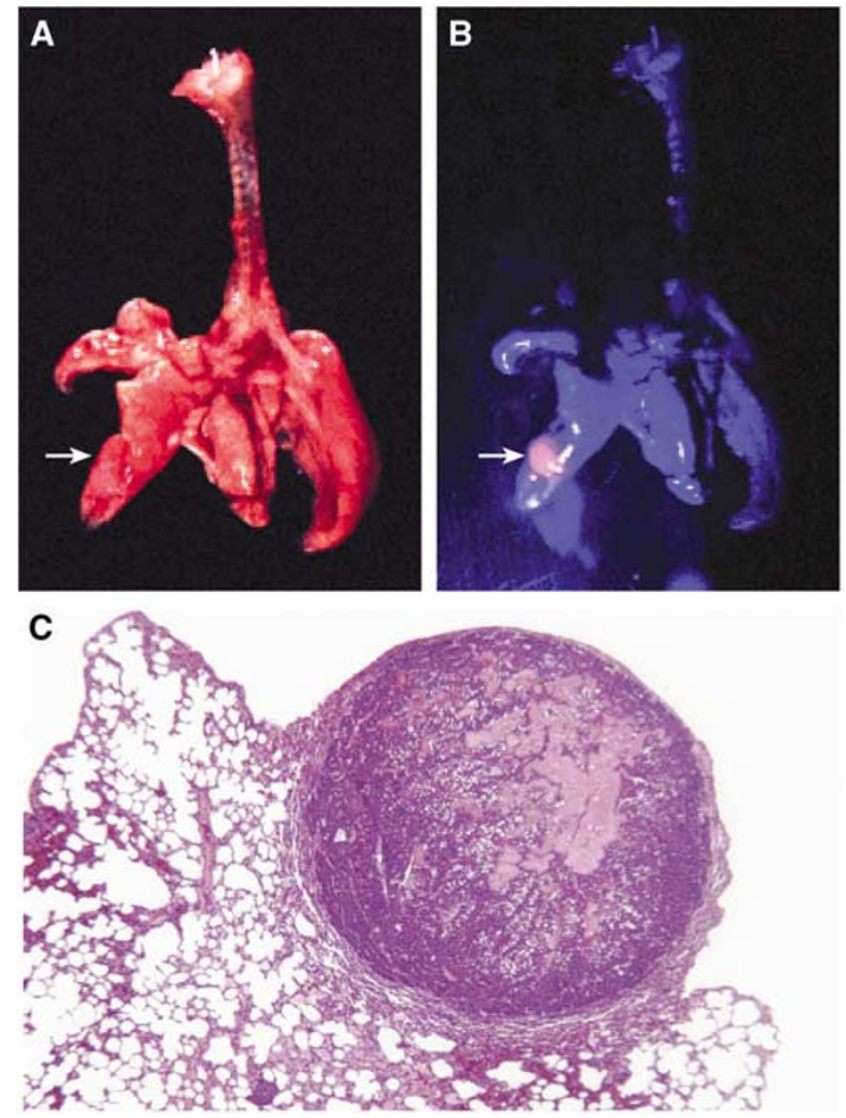

Figure 2 Photographic image of PpIX-stimulated red fluorescence in a metastatic tumour derived from a PyVT strain female (I5 weeks) I h following $200 \mathrm{mg} \mathrm{kg}^{-1}$ ALA administration. (A) Brightfield image of isolated bronchial tree. (B) Red fluorescence detection of a metastatic tumour in the lung (arrow). (C) Representative H\&E stained histological section of a tumour metastases from PyVT strain females $(4 \times)$.

surgery (Kriegmair et al, 1999; Holtl et al, 2001; Mayinger et al, 2001; Bogaards et al, 2005).

5-ALA-stimulated PpIX loading for use in PDD has shown great promise for human breast cancer, both in its application to define primary tumour margins during surgical removal, and for the identification of axillary lymph node metastases; (Ladner et al, 2001; Frei et al, 2004). We sought to examine the potential for 5ALA-stimulated PpIX loading in mammary tumours that develop in three well-characterised transgenic models of mammary tumorigenesis. The purpose of this investigation was three-fold: (1) to establish whether transgenic mouse mammary tumours are reliable models of human disease in terms of PpIX accumulation following 5-ALA administration, (2) to determine if the PpIX accumulation response was similar in each oncogenic model of tumorigenesis and (3) to develop an animal model for the study of the cellular and molecular mechanisms of enhanced PpIX accumulation. Our investigation revealed that rapid and significant PpIX loading occurred not only in primary tumours, but also in metastases and very early neoplastic lesions from the different transgenic models. We suggest that ALA-stimulated PpIX loading of early cancerous lesions of human mammary ducts will similarly contribute to early detection of human breast cancer.

\section{MATERIALS AND METHODS}

\section{Mice}

Three transgenic mouse models of mammary tumorigenesis were used for this investigation: (1) Transgenic strain FVB.Cg$\mathrm{Tg}$ (WapHRAS)69Lln Chr $\mathrm{Y}^{\mathrm{SJL}} / \mathrm{J}$ (hereafter referred to as HRAS) carries the human RAS oncogene (Harvey rat sarcoma viral oncogene homolog) with mammary tissue-specific gene expression driven by the Whey acidic protein (Wap) promoter (Andres et al, 1987). The HRAS strain represents a model for male mammary cancer, since solid adenosquamous mammary carcinomas develop only in male transgene carriers, given the integration of this transgene on the Y Chromosome. Care was taken to confirm that developing mammary tumours were not confused with salivary gland tumours that also develop in this strain. (2) The transgenic strain FVB/N-Tg(MMTV-PyVT)634Mul/J, (hereafter referred to as PyVT) carries the Polyoma Virus middle $\mathrm{T}$ antigen with mammary tissue-specific expression driven by the mouse mammary tumour virus (MMTV) promoter (Guy et al, 1992). Virgin females that carry the transgene develop poorly differentiated, multi-focal, invasive ductal carcinoma by 10-12 weeks of age, with a high incidence of lung metastases stemming from the primary mammary tumour (Lin et al, 2003). (3) The transgenic strain C57BL/6J-Tg(WapTAg) $1 \mathrm{Knw}$ (hereafter referred to as WapTag1) carries the Simian Virus 40 large $\mathrm{T}$ antigen (SV40 Tag) with mammary gland-restricted expression under control of the Wap promoter (Hüsler et al, 1998). Multiparous WapTag1 females develop late-onset mammary adenocarcinomas at 10-13 months of age predominantly of the papillary histological type, but may also show ductal, glandular or solid histology. Additional information about these mammary tumour mouse models can be obtained at the Mouse Tumor Biology Database (http://tumor. informatics.jax.org) via the Mouse Genome Informatics website (www.informatics.jax.org) of The Jackson Laboratory (Näf et al, 2002).

All mice were produced and housed in our research colony at The Jackson Laboratory under $14 \mathrm{~h}: 10 \mathrm{~h}$ light: dark cycles. Mice were provided with autoclaved NIH-31 diet (6\% fat, 19\% protein, vitamin and mineral fortified) (Purina Mills Intl., Brentwood MO) ad libitum and $\mathrm{HCl}$-acidified water $(\mathrm{pH} 2.8-3.2)$ ad libitum to retard bacterial growth. Animals were weaned at 18-23 days of age and housed in same sex groups of 3-5 in $51 \mathrm{in}^{2}\left(329 \mathrm{~cm}^{2}\right)$ polycarbonate cages containing sterilised White Pine shavings. All animal procedures were approved by the Animal Care and Use Committee of The Jackson Laboratory.

\section{5-ALA preparation and administration}

5-ALA.HCl (ASAT AG, Zug, Switzerland) was dissolved in sterile saline $(0.85 \% \mathrm{NaCl})$ immediately prior to administration, and kept in a light-protected vial. All animals were administered a tail vein injection of 5-ALA solution $(358 \mathrm{~mm})$ to achieve a final dose of $200 \mathrm{mg} \mathrm{kg}^{-1}$ of body weight. Animals were kept in their cages and maintained in a darkened room for a $60-75 \mathrm{~min}$ period prior to necropsy.

\section{Fluorescence detection}

Following in vivo incubation of 5-ALA, mice were euthanised by cervical dislocation or carbon dioxide asphyxiation. The 
exteriorised mammary glands were illuminated with blue light $(380-440 \mathrm{~nm}$ ) generated by a D-light system (Storz GmbH, Tuttlingen, Germany) to excite PpIX fluorescence. Digital images were recorded with a Leica DFX 350 camera mounted on a Wild M10 stereo-microscope (Leica Microsystems, Bannockburn, IL, USA) that was fitted with a $635 \mathrm{~nm}$ emission filter provided by Storz GmbH. Isolated lungs with mammary tumour metastasis were imaged with a $35 \mathrm{~mm}$ camera loaded with $160 \mathrm{~T}$ slide film (Eastman Kodak, Rochester, NY, USA), that was fitted with the $635 \mathrm{~nm}$ emission filter. When fluorescent tumour foci were $\leqslant 1 \mathrm{~mm}$ in size, blue background was digitally subtracted from the entire image to provide sufficient contrast for publication purposes (Adobe Photoshop CS, San Jose, CA, USA).

Isolated tumour foci were fixed overnight in Bouin's solution, and histological sections processed for hematoxylin and eosin (H\&E) staining. For early neoplastic lesions of WapTag1 mammary glands, a minimum of 30 serial sections were examined to confirm that no mature carcinomas were evident.

\section{Flow cytometry}

Mammary glands or palpable tumour foci from HRAS transgenic males and PyVT transgenic females and littermate controls were excised and minced with fine scissors in phosphate-buffered saline (PBS) containing $2 \mathrm{mg} \mathrm{ml}^{-1}$ collagenase type IA (Sigma, St. Louis, MO, USA). Isolated cell suspensions for flow cytometry were generated by a 15-min-incubation in collagenase/PBS under foil cover and disaggregration via successive aspiration through 18 and 20 gauge needles. The cell suspensions were kept on ice, under foil wrap until fluorescence measurements with a FACSVantage SE/ DiVa flow cytometer (BD Biosciences, San Jose, CA, USA). PpIX excitation was achieved with a $415.4 \mathrm{~nm}$ Innova 90-2 Krypton laser. PpIX fluorescence emissions were recorded following reflection by a $610 \mathrm{~nm}$ short pass dichroic mirror to a $590 \mathrm{~nm}$ long pass filter (Omega Optical Inc, Brattleboro, VT, USA). Acquistion and analysis of the data for 10000 tumour cells were performed with FACSDiva ver. 4.0, FACS Convert ver. 1.0 and CellQuestPro ver. 4.0.1 (BD Biosciences, San Jose, CA, USA).

\section{Statistical analysis}

The quantitative fluorescence flow cytometric data, representing a minimum of three independent replicate samples, was analysed by ANOVA with Statview software (SAS Institute Inc, Cary, NC, USA) with $P<0.05$ as the chosen level of statistical significance.
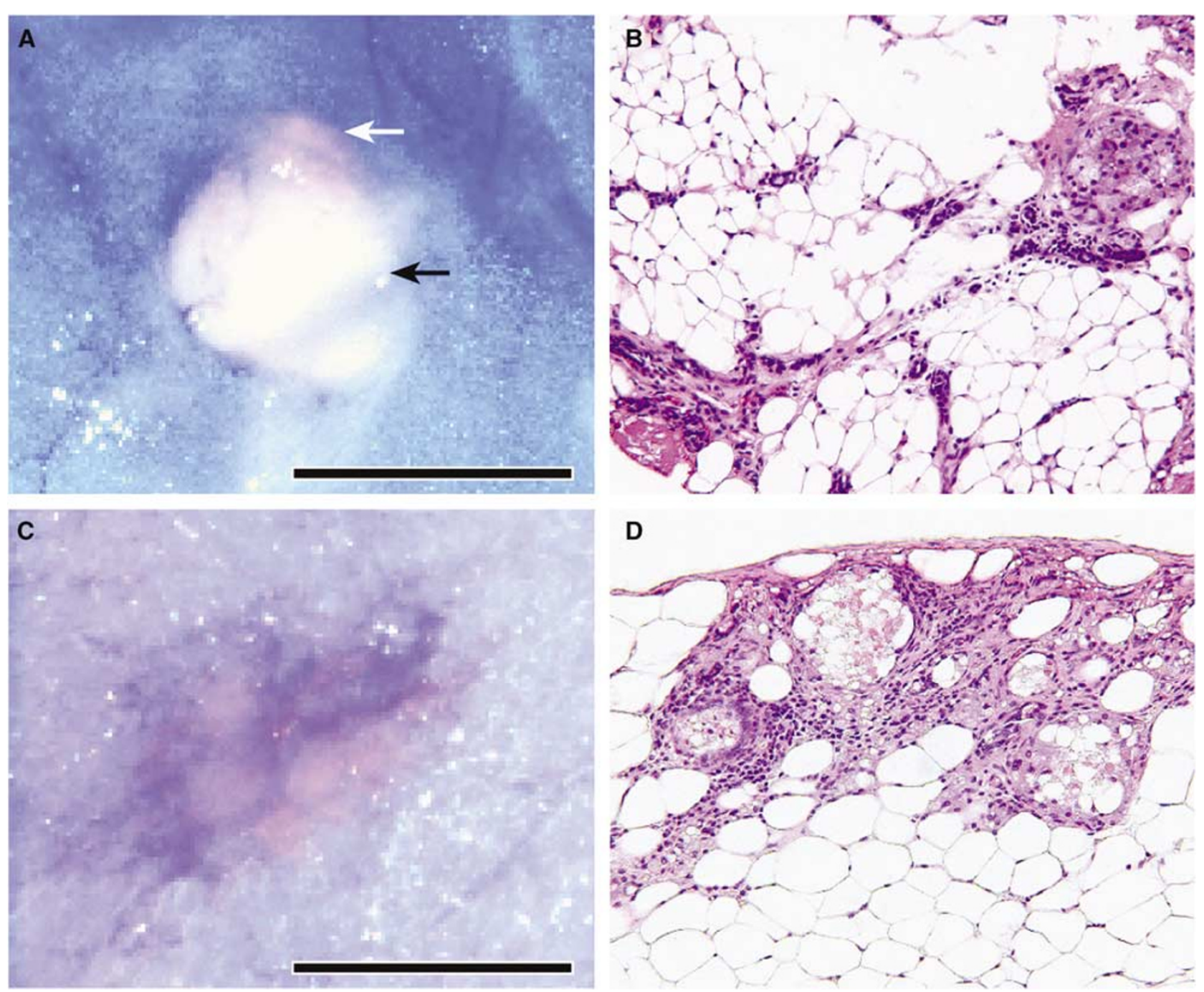

Figure 3 Digital image capture of PplX-stimulated red fluorescence in early DCIS lesions of two WapTag I females (4I and 45 weeks) I h following in vivo administration of $200 \mathrm{mg} \mathrm{kg}^{-1}$ ALA. (A, C) Red fluorescence detection of DCIS in the mammary gland. In (A) The white arrow indicates the region of DCIS and the black arrow indicates accumulated proteinaceous fluid. (B, D) Corresponding H\&E stained histological sections $(20 \times)$. Scale bar represents $0.5 \mathrm{~mm}$. 


\section{RESULTS}

Mice bearing palpable mammary tumours were investigated for their PpIX accumulation response following 5-ALA administration. Mice were euthanised and the mammary tumours exteriorised to examine PpIX accumulation under blue light excitation. Mammary tumours in each of the transgenic models responded to 5-ALA administration with significant PpIX accumulation, as measured by tumour-selective red fluorescence relative to the normal surrounding tissues. Fluorescence was most pronounced in PyVT mammary tumours, but was clearly evident in tumours from each transgenic model, despite differences in histological subtype and the gender of the tumour-bearing animal (Table 1, Figure 1). WapTag1 tumours present with the most varied histology, but prominent PpIX fluorescence was observed for tumours of both papillary (Figure 1A and B) and glandular-to-solid subtypes. In HRAS males, tumours 1-2 mm in diameter were easily recognised by red fluorescent emissions following 5-ALA treatment, with similar solid-type histological characteristics as the larger tumour masses (Figure $1 \mathrm{C}$ and D). In young PyVT females, the multiplicity of tumour initiation within a single mammary gland was evidenced by red fluorescence following 5-ALA administration, with some of the foci measuring only $0.1 \mathrm{~mm}$ (Figure $1 \mathrm{E}$ and F). Primary tumours arising in PyVT transgene females frequently metastasize to the lungs. Figure 2 shows an isolated bronchial tree with lung metastases removed from a PyVT strain female. Under blue light, the metastatic focus shines bright red relative to the normal lung tissue, showing that PpIX synthesis remains upregulated in tumour cells with metastatic competence.

Given the evidence for PpIX accumulation in palpable, mature mammary tumours, we administered 5-ALA to WaptTag1 transgenic female mice prior to the anticipated age for palpable tumour presentation, when early stages of tumour progression are predicted for this mouse model. In four independent WapTag1 females, 5-ALA stimulated PpIX accumulation permitted red fluorescence detection of areas histologically confirmed as ductal carcinoma in situ (DCIS) with accompanying inflammation. Two examples of PpIX stimulated red fluorescence associated with DCIS are illustrated in Figure 3. In Figure 3A, the fluorescent region of DCIS is adjacent to an accumulation of proteinaceous fluid, suggesting the early tumour has stopped the flow of fluids through the duct, while in Figure $3 \mathrm{C}$, the fluorescent region is well demarcated from the surrounding normal gland. Detection of DCIS by PpIX fluorescence indicates that an enhanced capacity to synthesise PpIX is a very early characteristic in the neoplastic transition of these mammary tumours.

PpIX-stimulated fluorescence was quantified by flow cytometric analysis in palpable (5-10 $\mathrm{mm}$ diameter) homogeneous mammary tumours of HRAS transgenic males and PyVT transgenic females. A comparison of the fluorescence emitted from isolated HRAS tumour cells derived from 5-ALA-primed vs control tumourbearing males is shown in Figure 4A: the HRAS tumours exposed to 5-ALA had a significant, five-fold increase in fluorescence emissions. Similarly, the mammary tumour cells isolated from PyVT strain females primed with 5-ALA showed a significant, seven-fold increase in fluorescence emissions compared to tumour cells obtained from control animals (Figure 4B). To illustrate the significant difference in the 5-ALA response of mammary tumors $v s$ the normal surrounding tissues, we also compared the PpIX fluoresence emissions of normal mammary glands obtained from nontransgene carrier, virgin littermates of the PyVT females. Like the tumour cells, the normal mammary glands were either exposed to 5-ALA $\left(200 \mathrm{mg} \mathrm{kg}^{-1}\right)$ for $1 \mathrm{~h}$ in vivo or were obtained directly from unprimed control females. 5-ALA administration induced a significant, two-fold increase in red fluorescence emissions in the normal mammary gland, although this increase was significantly less than the seven-fold increase observed in 5-ALA-primed PyVT tumour cells, and did not interfere with the visual margins of differential red fluorescence observed between tumour cells and normal tissues in the intact animal (Figure 1E).

\section{DISCUSSION}

To establish whether transgenic mouse models of mammary tumorigenesis are relevant models for human breast cancer in terms of their response to 5-ALA, we have evaluated three wellcharacterised transgenic strains that develop mammary tumours under powerful oncogenic stimulation - HRAS, PyVT and SV40 Tag - for their ability to accumulate PpIX following administration of 5-ALA. Our findings showed that the mammary tumours from each transgenic strain rapidly and selectively converted 5-ALA to PpIX, and showed five- to seven-fold enhanced fluorescence compared with surrounding normal tissues under blue light excitation. Similar to the primary mammary tumours, spontaneous metastatic lesions to the lungs of tumour bearing PyVT mice were readily demarcated by PpIX accumulation and red fluorescence under blue light, suggesting that the cellular mechanisms responsible for PpIX accumulation are retained within tumour populations that acquire metastatic competence. This follows the findings of Frei et al (2004) who found that metastatic breast cancer cells in axillary lymph nodes of human patients could be identified by PpIX accumulation.

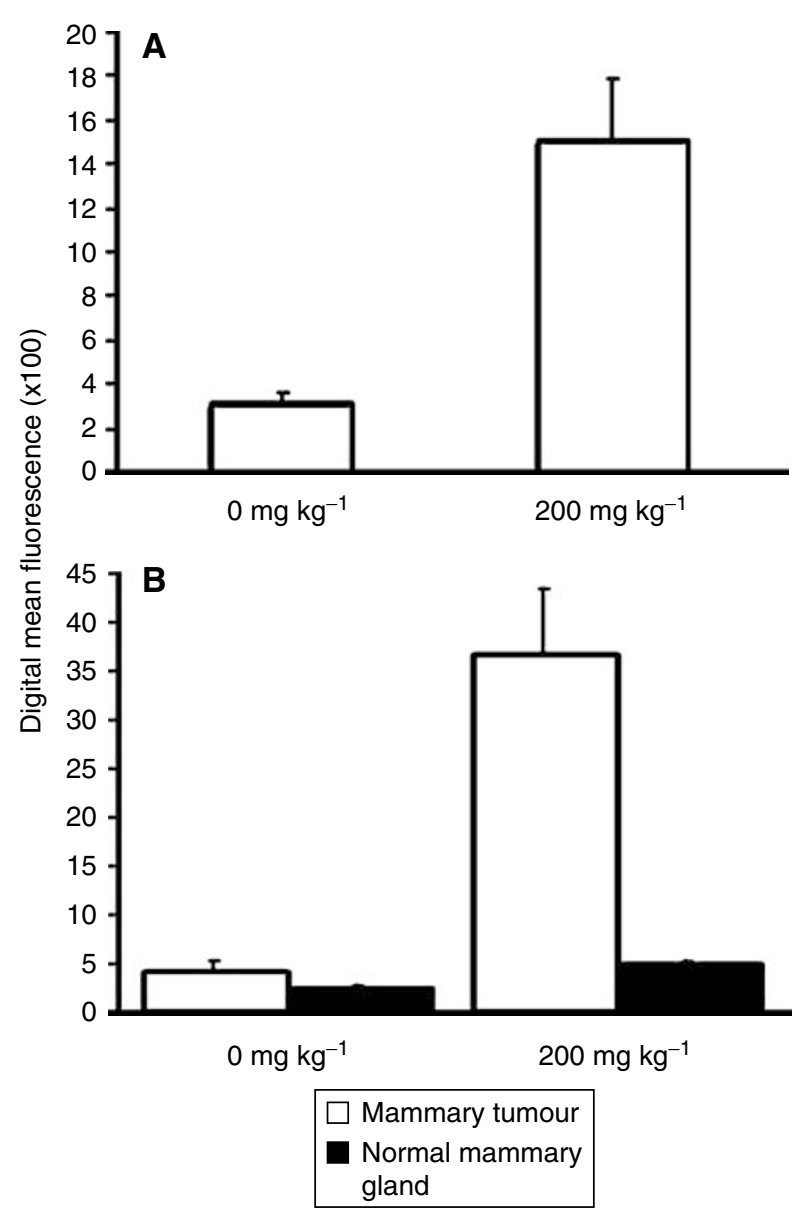

Figure 4 Flow cytometric measurement of digital mean fluorescence in PplX-loaded tumour cells $\mathrm{I}$ h following in vivo administration of $200 \mathrm{mg} \mathrm{kg}^{-1}$ ALA (mean \pm s.e.). (A) Mammary tumour cells from HRAS males. (B) Normal mammary gland and mammary tumour cells from PyVT females. 
PpIX accumulation in the primary tumours of each strain was consistent, despite fundamental differences in histological subtype, latency of onset, metastatic potential and gender of the tumourbearing host. Thus, the transgenic models parallel the findings for human breast cancer cases in that the PpIX accumulation phenotype is not selective for any particular tumour histological subtype (Ladner et al, 2001). Most significantly, our observations of PpIX accumulation in early DCIS of the WapTag1 transgenic mammary glands also suggested this phenotypic switch is an early event in the tumorigenic process. If enhanced PpIX synthesis can similarly be utilized as an early marker for human breast cancer, the impetus is strong to develop noninvasive imaging strategies that take advantage of tumour cell-selective changes in PpIX biochemistry for early detection.

A new avenue of clinical tumour imaging for human breast cancer has opened with the availability of fiberoptic ductoscopy (Mokbel et al, 2005; Sauter et al, 2005). Endoscopes of fine diameter $(<1 \mathrm{~mm})$ inserted through the breast nipple can gather three-dimensional structural information of the mammary ducts, and are under investigation as a tool for breast cancer detection. The high quality of optical imaging allows localisation of micro-pathology, such as DCIS of the epithelial lining within the mammary ducts. Our findings for enhanced PpIX accumulation in early DCIS of mouse mammary tumours suggests that fiberoptic ductoscopy combined with a fluorescence detection system for PpIX could increase the specificity and sensitivity of ductoscopy for early tumour detection in the human breast.
Confirmation that mouse models of both male and female mammary tumorigenesis parallel the clinical findings for human breast cancer in terms of PpIX synthesis could lead to new lines of investigation for the detection, diagnosis and therapy of breast cancer. One advantage of the mouse models of mammary tumorigenesis over cell culture models is that cellular events leading to the PpIX accumulation phenotype can be genetically and chronologically mapped according to the various stages of tumorigenesis. Furthermore, an investigation as to whether altered regulation of the haeme synthesis pathway is important for tumour development can be further addressed in genetically engineered mouse models of mammary tumorigenesis.

\section{ACKNOWLEDGEMENTS}

We thank ASAT AG for supplying 5-ALA, Storz GmbH for the Dlight equipment and optical filters; Greg Martin, Jim Denegre, Jim Miller, Jennifer Torrance and Sarah Williamson for photographic and digital imaging assistance; Bonnie Lyons for technical assistance, and Igor Mikaelian for help with pathological assessments. Thanks to Drs John Sundberg and Barbara Knowles for reading the manuscript and making helpful suggestions. This study was supported by the National Cancer Institute Cancer Core Grant CA-34196 (to The Jackson Laboratory, TMD), an anonymous foundation (AMD, WGB), the Maine Cancer Foundation (AMD), the National Science Foundation IGERT Program (0221625) (KSF), and the University of Zurich (HW).

\section{REFERENCES}

af Klinteberg C, Enejder A, Wang I, Andersson-Engels S, Svanberg S, Svanberg K (1999) Kinetic fluorescence studies of 5-aminolevulinic acidinduced protoporphyrin IX accumulation in basal cell carcinomas. J Photochem Photobiol B 49: 120-128

Andres A, Schonenberger C, Groner B, Hennighausen L, LeMeur M, Gerlinger P (1987) Ha-ras oncogene expression directed by a milk protein gene promoter: tissue specificity, hormonal regulation, and tumor induction in transgenic mice. Proc Natl Acad A Sci USA 84: 1299- 1303

Bedwell J, MacRobert A, Phillips D, Bown S (1992) Fluorescence distribution and photodynamic effect of ALA-induced PPIX in the DMH rat colonic tumour model. Br J Cancer 65: 818-824

Betz C, Stepp H, Janda P, Arbogast S, Grevers G, Baumgartner R, Leunig A (2002) A comparative study of normal inspection, autofluorescence and 5-ALA-induced PPIX fluorescence for oral cancer diagnosis. Int J Cancer 97: $245-252$, doi:10.1002/ijc/.1596

Bogaards A, Aalders M, Zeyl C, de Blok S, Dannecker C, Hillemanns P, Stepp H, Sterenborg H (2002) Localization and staging of cervical intraepithelial neoplasia using double ratio fluorescence imaging. J Biomed Opt 7: 215-220

Bogaards A, Varma A, Zhang K, Zach D, Bisland S, Moriyama E, Lilge L, Muller P, Wilson B (2005) Fluorescence image-guided brain tumour resection with adjuvant metronomic photodynamic therapy: pre-clinical model and technology development. Photochem Photobiol Sci 4: 438-442

Casas A, Fukuda H, Di Venosa G, Batlle A (2001) Photosensitization and mechanism of cytotoxicity induced by the use of ALA derivatives in photodynamic therapy. $B r$ J Cancer 85: 279-284, doi:10.1054/bjoc. 2001.1875

Chen J, Mak N, Cheung N, Leung R, Peng Q (2001) Endogenous production of protoporphyrin IX induced by 5 -aminolevulinic acid in leukemia cells. Acta Pharmacol Sin 22: $163-168$

Collaud S, Juzeniene A, Moan J, Lange N (2004) On the selectivity of 5aminolevulinic acid-induced protoporphyrin IX formation. Curr Med Chem Anti-Canc Agents 4: 301-316

Frei K, Bonel H, Frick H, Walt H, Steiner R (2004) Photodynamic detection of diseased axillary sentinel lymph node after oral application of aminolevulinic acid in patients with breast cancer. $\mathrm{Br} J$ Cancer 90: $805-809$, doi:10.1038/sj.bjc.6601615
Guy C, Cardiff R, Muller W (1992) Induction of mammary tumors by expression of polyomavirus middle $\mathrm{T}$ oncogene: A transgenic model for metastatic disease. Mol Cell Biol 12: 954-961

Hinnen P, de Rooij F, van Velthuysen M, Edixhoven A, van Hillegersberg R, Tilanus H, Wilson J, Siersema P (1998) Biochemical basis of 5-aminolaevulinic acid-induced protoporphyrin IX accumulation: a study in patients with (pre)malignant lesions of the oesophagus. $\mathrm{Br} J$ Cancer 78: 679-682

Holtl L, Eder I, Klocker H, Hobisch A, Bartsch G, Stenzl A (2001) Photodynamic diagnosis with 5-aminolevulinic acid in the treatment of secondary urethral tumors: first in vitro and in vivo results. Eur Urol 39: $178-182$

Hua Z, Gibson S, Foster T, Hilf R (1995) Effectiveness of deltaaminolevulinic acid-induced protoporphyrin as a photosensitizer for photodynamic therapy in vivo. Cancer Res 55: 1723-1731

Hüsler M, Kotopoulis K, Sundberg J, Tennent B, Kunig S, Knowles B (1998) Lactation-induced WAP-SV40 Tag transgene expression in C57BL/6J mice leads to mammary carcinoma. Transgenic Res 7: $253-263$

Krieg R, Messmann H, Rauch J, Seeger S, Knuechel R (2002) Metabolic characterization of tumor cell-specific protoporphyrin IX accumulation after exposure to 5-aminolevulinic acid in human colonic cells. Photochem Photobiol 76: 518-525

Kriegmair M, Zaak D, Knuechel R, Baumgartner R, Hofstetter A (1999) 5-Aminolevulinic acid-induced fluorescence endoscopy for the detection of lower urinary tract tumors. Urol Int 63: 27-31

Ladner D, Steiner R, Allemann J, Haller U, Walt H (2001) Photodynamic diagnosis of breast tumours after oral application of aminolevulinic acid. Br J Cancer 84: 33 -37, doi:10.1054/bjoc.2000.1532

Lin E, Jones J, Li P, Zhu L, Whitney K, Muller W, Pollard J (2003) Progression to malignancy in the Polyoma Middle T oncoprotein mouse breast cancer model provides a reliable model for human diseases. Am J Pathol 163: 2113-2126

Löning M, Diddens H, Küpker W, Diedrich K, Hüttmann G (2004) Laparoscopic fluorescence detection of ovarian carcinoma metastases using 5-aminolevulinic acid-induced protoporphyrin IX. Cancer 100: 1650 - 1656, doi:10.1002/cncr.20155 
Mayinger B, Neidhardt S, Reh H, Martus P, Hahn E (2001) Fluorescence induced with 5-aminolevulinic acid for the endoscopic detection and follow-up of esophageal lesions. Gastrointest Endosc 54: $572-578$

Moesta K, Ebert B, Handke T, Nolte D, Nowak C, Haensch W, Pandrey R, Dougherty T, Rinneberg H, Schlag P (2001) Protoporphyrin IX occurs naturally in colorectal cancers and their metastases. Cancer Res 61: 9 $91-999$

Mokbel K, Escobar P, Matsunaga T (2005) Mammary ductoscopy: current status and future prospects. Eur J Surg Oncol 31: 3-8, doi:10.1016/ j.ejso.2004.10.004

Näf D, Krupke D, Sundberg J, Eppig J, Bult C (2002) The mouse tumor biology database: a public resource for cancer genetics and pathology of the mouse. Cancer Res 62: $1235-1240$

Navone N, Polo C, Frisardi A, Andrade N, Batlle A (1990) Heme biosynthesis in human breast cancer - mimetic 'in vitro' studies ad soe heme enzymic activity levels. Int J Biochem 22: 1407-1411

Olivo M, Lau W, Manivasager V, Hoon T, Christopher C (2003) Fluorescence confocal microscopy and image analysis of bladder cancer using 5-aminolevulinic acid. Int J Oncol 22: 523-528

Otake M, Nishiwaki M, Kobayashi Y, Baba S, Kohno E, Kawasaki T, Fujise Y, Nakamura H (2003) Selective accumulation of ALA-induced PpIX and photodynamic effect in chemically induced heptocellular carcinoma. $\mathrm{Br} J$ Cancer 89: 730 - 736, doi:10.1038/sj.bjc.6601135

Peng Q, Moan J, Warloe T, Nesland J, Rimington C (1992) Distribution and photosensitizing efficiency of porphyrins induced by application of exogenous 5-aminolevulinic acid in mice bearing mammary carcinoma. Int J Cancer 52: $433-443$

Ponka P (1999) Cell biology of heme. Am J Med Sci 318: 241-256

Sauter E, Ehya H, Klein-Szanto A, Wagner-Mann C, MacGibbon B (2005) Fiberoptic ductoscopy findings in women with and without spontaneous nipple discharge. Cancer 103: 914 -921, doi 10.1002/cncr.20865

Stepinac T, Felley C, Jornod P, Lange N, Gabrecht T, Fontolliet C, Grosjean $P$, vanMelle $G$, van den Bergh $H$, Monnier P, Wagnieres G, Dorta G (2003) Endoscopic fluorescence detection of intraepithelial neoplasia in Barrett's esophagus after oral administration of aminolevulinic acid. Endoscopy 35: $663-668$

Stummer W, Stocker S, Wagner S, Stepp H, Fritsch C, Goetz C, Goetz A, Kiefmann R, Reulen H (1998) Intraoperative detection of malignant gliomas by 5 -aminolevulinic acid-induced porphyrin fluorescence. Neurosurgery 42: $518-525$

Van Hillegersberg R, Van den Berg J, Kort W, Terpstra O, Wilson J (1992) Selective accumulation of endogenously produced porphyrins in a liver metastasis model in rats. Gastroenterology 103: 647-651 